\title{
Damages to return with a possible occurrence of eco-technology innovation measured by DEA environmental assessment
}

Toshiyuki Sueyoshi

\author{
*Correspondence: \\ Toshiyuki.Sueyoshi@nmt.edu \\ Department of Management, \\ New Mexico Institute \\ of Mining and Technology, \\ 801 Leroy Place, Socorro, NM \\ 87801, USA
}

\begin{abstract}
Environmental assessment and pollution protection are important concerns in modern business and policy. Consumers are interested in corporate efforts on not only their products but also environmental protections and pollution preventions. To attain a high level of sustainability (i.e., economic success and pollution prevention), all entities in private and public sectors need to pay attention to green technology innovation. This study considers that eco-technology innovation can combat the global warming and climate change, which the world is now facing as a major policy issue, along with its related business and policy supports. It is clear that any engineering and science efforts cannot attain any policy goal on the climate change without supports from social science perspectives, including business and economics. An important concern discussed in this study is how to identify an effective decision on eco-technology innovation and its influence on sustainability. To discuss the global issue at the world level, this study is first interested in measuring the performance of their operational and environmental achievements and then pays attention to Damages to Return (DTR). The economic concept indicates a level of change of undesirable outputs (e.g., $\mathrm{CO}_{2}$ ) by increasing one unit of a desirable output (e.g., oil production). To assess the magnitude of DTR, this study proposes a use of Data Envelopment Analysis (DEA). The proposed DEA assessment theoretically provides corporate leaders and policy makers with information regarding how to invest in eco-technology innovation for abatement of undesirable outputs, so enhancing the level of corporate or social sustainability.
\end{abstract}

Keywords: Environment, Eco-technology innovation, Sustainability, Data Envelopment Analysis

\section{Introduction}

The Intergovernmental Panel on Climate Change (IPCC 2014), established by United Nations (UN) environmental program, has reported the policy suggestion that it is necessary for us to reduce an amount of greenhouse gas (GHG) emissions, in particular $\mathrm{CO}_{2}$, by $40-70 \%$ (compared with 2010) until 2050 and to the level of almost zero by the end of the twenty-first century via shifting our current systems to energy-efficient ones. The challenge on the climate change makes the conventional profit-driven business logic and practice inappropriate and incompatible with a worldwide trend toward a sustainable society. It is necessary for firms to change their business operations to adapt to various regulations for GHG emission reduction.

(c) The Author(s) 2017. This article is distributed under the terms of the Creative Commons Attribution 4.0 International License (http://creativecommons.org/licenses/by/4.0/), which permits unrestricted use, distribution, and reproduction in any medium, provided you give appropriate credit to the original author(s) and the source, provide a link to the Creative Commons license, and indicate if changes were made. 
Although the scientific claim proposed by the IPCC is very important, this study can easily imagine that no organization at the level of nations and companies pay serious attention the claim, because the IPCC within the UN does not have any clear implementation strategy from business and policy perspectives. We also combat not only the global warming and climate change but also other types of various pollutions (e.g., air, water and nuclear waste pollutions). It may be true that all the entities (i.e., nations and companies) prefer the development of their clean images, indeed, so that they can attain their economic prosperities. The reality may be different from the truth. Just a green image is important for them. A typical example can be found in the recent corporate scandal of Volkswagen in 2015 that has been long cheating on the $\mathrm{CO}_{2}$ emissions produced by cars. See also the new Repablican goverment starts neglecting the international agreement in the United States.

The purpose of this study is to conceptually and theoretically examine the corporate sustainability (i.e., economic success and pollution prevention at the corporate level) from Damages to Return (DTR) where DTR indicates a level of change on undesirable outputs (e.g., $\mathrm{CO}_{2}$ ) by increasing one unit of a desirable output (e.g., unit production).

The goal to establish a sustainable society requires a new methodology that can integrate the companies' environmental measures with their operational measures in such a manner that it provides a holistic assessment for overall performance. This study proposes a usage of Data Envelopment Analysis (DEA) as a practical approach to evaluate the unified (operational and environmental) performance of private and public organizations from the perspective of corporate sustainability. The proposed DEA approach has several advantages over existing methods in production economics. First, it incorporates four disposability concepts such as natural disposability and managerial disposability as well as these conceptual combinations. Outputs and inputs, characterizing companies' operational and environmental performance, are separated under these disposability concepts. Moreover, the proposed approach can identify effective investment opportunities for GHG abatement by utilizing the concept of congestion on undesirable outputs. This study summarizes both business and policy implications obtained from the proposed DEA application.

All abbreviations and nomenclatures used in this study are summarized as follows: DC: Desirable Congestion, DEA: Data Envelopment Analysis, DMU: Decision Making Unit, DTS: Damages to Scale, DTR: Damages to Return, RTS: Returns to Scale, RTD: Returns to Damage, UC: Undesirable Congestion, URS: Unrestricted, UEN: Unified Efficiency under Natural disposability, UEM: Unified Efficiency under Managerial disposability, $X$ : a column vector of $m$ inputs, $G$ : a column vector of $s$ desirable outputs, $B$ : a column vector of $h$ undesirable outputs, $d_{i}^{x}$ : an unknown slack variable of the $i$ th input, $d_{r}^{g}$ : an unknown slack variable of the $r$ th desirable output, $d_{f}^{b}$ : an unknown slack variable of the $f$ th undesirable output, $\lambda$ : an unknown column vector of intensity (or structural) variables, $R_{i}^{x}$ : a data range related to the $i$ th input, $R_{r}^{g}$ : a data range related to the $r$ th desirable output, $R_{f}^{b}$ : a data range related to the fth undesirable output, $v_{i}$ : a dual variable of the $i$ th input, $u_{r}$ : a dual variable of the $r$ th desirable output, $w_{f}$ : a dual variable of the $f$ th undesirable output and $\sigma$ : a dual variable to indicate the intercept of a supporting hyperplane on production and pollution possibility set.

The remainder of this study is organized as follows. Section 2 summarizes previous research efforts on DEA applied to energy and environment. Section 3 discusses underlying concepts incorporated into the proposed DEA environmental assessment. 
Section 4 describes mathematical models under natural and managerial disposability. Section 5 discusses how to incorporate a possible occurrence of desirable congestion, or eco-technology innovation, into the proposed DEA formulations. Section 6 concludes this research along with future extensions.

\section{Previous research}

The literature study on DEA can be found in Glover and Sueyoshi (2009) and Ijiri and Sueyoshi (2010). As an extension of these works, the systematic literature survey on DEA applied to energy and environment can be found in Sueyoshi and Yuan (2016a, b), Sueyoshi et al. (2017) and Sueyoshi and Goto (2017). Since the three studies provide us with detailed descriptions on the literature, this study does not need to discuss previous works on such DEA applications in this section, except specifying that the energybased classification on 693 articles may be separated into primary and secondary energy sources as proposed in Sueyoshi et al. (2017). The purpose of this study is to add a theoretical aspect on DEA environmental assessment to the research of Sueyoshi et al. (2017) from the perspective of eco-technology innovation and/or a managerial challenge.

\section{Underlying concepts for disposability}

In order to describe mathematical formulations for DEA environmental assessment, this study needs to introduce concepts concerning "natural disposability" and "managerial disposability." To describe them more clearly, let us consider $X \in R_{+}^{m}$ as an input vector with $m$ components, $G \in R_{+}^{s}$ as a desirable output vector with $s$ components and $B \in R_{+}^{h}$ as an undesirable output vector with $h$ components. In these column vectors, the subscript $(j)$ is used to stand for the $j$ th $\mathrm{DMU}$, whose vector components are strictly positive.

Using an axiomatic expression, this study specifies a unified (operational and environmental) production possibility set to express natural $(N)$ and that for managerial $(M)$ disposability by the two types of output vectors and an input vector, respectively, as follows:

$$
\begin{aligned}
& P_{v}^{N}(X)=\left\{(G, B): G \leq \sum_{j=1}^{n} G_{j} \lambda_{j}, B \geq \sum_{j=1}^{n} B_{j} \lambda_{j}, X \geq \sum_{j=1}^{n} X_{j} \lambda_{j}, \sum_{j=1}^{n} \lambda_{j}=1 \& \lambda_{j} \geq 0(j=1, \ldots, n)\right\} \text { and } \\
& P_{v}^{M}(X)=\left\{(G, B): G \leq \sum_{j=1}^{n} G_{j} \lambda_{j}, B \geq \sum_{j=1}^{n} B_{j} \lambda_{j}, X \leq \sum_{j=1}^{n} X_{j} \lambda_{j}, \sum_{j=1}^{n} \lambda_{j}=1 \& \lambda_{j} \geq 0(j=1, \ldots, n)\right\} .
\end{aligned}
$$

$P_{v}^{N}(X)$ stands for a production and pollution possibility set under natural $(N)$ disposability, meanwhile $P_{v}^{M}(X)$ is that of managerial disposability. The subscript $(v)$ stands for "variable" RTS or DTS because the constraint $\left(\sum_{j=1}^{n} \lambda_{j}=1\right)$ is incorporated into the two axiomatic expressions.

The difference between the two disposability concepts is that the production technology under natural disposability, or $P_{v}^{N}(X)$, has $X \geq \sum_{j=1}^{n} X_{j} \lambda_{j}$, implying that a DMU can attain an efficiency frontier by reducing a directional vector of inputs. Meanwhile, that of the managerial disposability, or $P_{v}^{M}(X)$, has $X \leq \sum_{j=1}^{n} X_{j} \lambda_{j}$, implying that a DMU can attain a status of an efficiency frontier by increasing a directional vector of inputs. Meanwhile, a common feature of the two disposability concepts is that both have $G \leq \sum_{j=1}^{n} G_{j} \lambda_{j}$ and $B \geq \sum_{j=1}^{n} B_{j} \lambda_{j}$ in their axiomatic expressions. These conditions intuitively appeal to us because an efficiency frontier for desirable outputs should locate 
above or on all observations, while that of undesirable outputs should locate below or on these observations. See Porter and van der Linde (1995) for a detailed description concerning why we need the managerial disposability in terms of enhancing corporate sustainability.

By changing these axiomatic structures by dropping $\sum_{j=1}^{n} \lambda_{j}=1$, the production and pollution possibility set becomes as follows:

$$
\begin{aligned}
& P_{c}^{N}(X)=\left\{(G, B): G \leq \sum_{j=1}^{n} G_{j} \lambda_{j}, B \geq \sum_{j=1}^{n} B_{j} \lambda_{j}, X \geq \sum_{j=1}^{n} X_{j} \lambda_{j} \& \lambda_{j} \geq 0(j=1, \ldots, n)\right\} \text { and } \\
& P_{c}^{M}(X)=\left\{(G, B): G \leq \sum_{j=1}^{n} G_{j} \lambda_{j}, B \geq \sum_{j=1}^{n} B_{j} \lambda_{j}, X \leq \sum_{j=1}^{n} X_{j} \lambda_{j} \& \lambda_{j} \geq 0(j=1, \ldots, n)\right\},
\end{aligned}
$$

where the two equations $\operatorname{drop} \sum_{j=1}^{n} \lambda_{j}=1$ from their axiomatic structures. The subscript $(c)$ is used to express constant RTS or DTS.

Here, it is necessary to discuss that an input vector is usually assumed to project toward a decreasing direction in the previous research efforts on conventional DEA and production economics. The assumption is often inconsistent with the reality related to environmental protection. For example, many governments and firms consider an increase in input resources to yield an annual "growth" on desirable outputs. Thus, the conventional framework of DEA is not consistent with the economic expansion, or "economic growth," because the previous DEA studies have implicitly assumed the minimization of total production cost. The cost concept may be acceptable for performance analysis under "economic recession" or "stagnation," but not in many cases where industrial planning and corporate strategy are based upon their economic growths. Thus, it is easily imagined that DEA environmental assessment is conceptually different from the conventional use of DEA. The cost concept for guiding public and private entities in their strategy development is average cost (under constant RTS) or marginal cost (under variable RTS), not the total cost, anymore. Furthermore, an opportunity cost, originated from business risk due to industrial pollutions and the other types of various environmental problems (e.g., the nuclear power plant accident at Fukushima Daiichi in Japan), has a major role in modern corporate governance issues. Such cost concepts for current policy making and modern business are implicitly incorporated in formulating the two disposability concepts, in particular in managerial disposability.

Figure 1, obtained from Sueyoshi and Goto (2017), depicts the analytical structure for measuring unified efficiency. For our visual convenience, Fig. 1 considers the achievement, listed as $K$, of a DMU that uses a single component of an input vector ( $x$ in the horizontal axis) to produce both a single component of a desirable output vector ( $g$ on the vertical axis) and a single component of an undesirable output vector ( $b$ on the vertical axis). The description can be easily extendable to the case of multiple components of the three vectors in the proposed DEA formulations.

Here, let us consider that the achievement of the $k$ th DMU is specified as $\left(x_{i k}, g_{r k}, b_{f k}\right)$ in Fig. 1. To measure its unified efficiency, we need to incorporate the two efficiency frontiers into the proposed analytical structure, as visually described in Fig. 1. One of the two frontiers is an efficiency frontier for a desirable output. The frontier is listed as a top frontier in Fig. 1. The other (a bottom frontier) is an efficiency frontier for an undesirable 


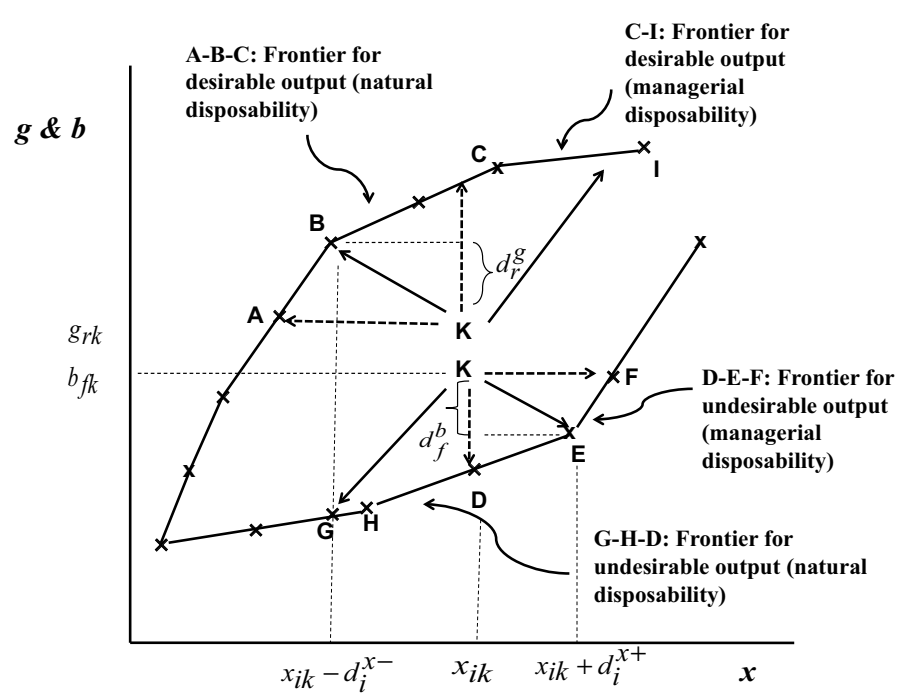

Fig. 1 Two efficiency frontiers for measuring unified efficiency. (a) The direction of NW (North-West) indicates a projection of $\mathrm{K}$ toward an efficiency frontier $(A-B-C)$ that is incorporated into the conventional framework of DEA. (b) DEA environmental assessment incorporates the projection of SW (South-West) from $K$ toward an efficiency frontier $(G-H-D)$ under natural disposability and the projection of SE (South-East) from $K$ toward an efficiency frontier ( $D-E-F)$ under managerial disposability. The projection of NW serves as a basis for the performance assessment of conventional DEA. The projection of NE (North-East) toward an efficient frontier $(C-I)$ has been excluded from the conventional use. The proposed environmental assessment incorporates the projection toward NE along with an increase in components of an input vector. (c) NW + SW: natural disposability and NE + SE: managerial disposability. Both are incorporated in the proposed DEA environmental assessment. The two disposability concepts make a use of DEA different from the conventional use (by only NW direction for operational performance enhancement)

output. The efficiency frontier on the undesirable output is separated into the frontier under natural disposability (the left-hand side of the efficiency frontier for the undesirable output) and the frontier under managerial disposability (the right-hand side of the efficiency frontier for the undesirable output). As depicted in Fig. 1, DEA formulations proposed in this study do not incorporate the assumption on by-products on undesirable outputs. Hereafter, this study follows their description of Sueyoshi and Goto (2017). $N W$ (North West) projections to enhance operational performance Let us consider the $k$ th DMU whose performance is measured on the combination between an input and a desirable output as depicted in Fig. 1. If the $k$ th DMU is inefficient in operational performance, then it needs to improve its operational efficiency by increasing the amount of a desirable output, expressed by $d_{r}^{g}$, and/or decreasing the amount of an input, expressed by $d_{i}^{x-}$. A possible projection can be found on $B$ as an example. A piece-wise linear contour line ( $A-$ $B-C$ ) indicates an efficiency frontier where $K$ needs to be projected. The direction of such a possible projection toward $A-B-C$ should be expressed by both the sign of an input-related slack and that of a desirable output-related slack in Model (1). In this study, the direction of projection belongs to "NW" which indicates the enhancement of operational efficiency.

NE (North East) projections to enhance operational performance The projection of NE (North-East) has been excluded from the conventional use. The proposed environmental assessment incorporates the type of projections toward NE, along with an input increase, in Fig. 1. Here, it is important to note that this type of projections by an input vector increase has been never discussed in previous DEA studies. 
SW (South West) projections to enhance environmental performance under natural disposability Fig. 1 visually describes natural disposability in the input (on a horizontal coordinate) and the undesirable output (on a vertical coordinate) whose projections are directed toward SW (South West). One of such directions is the projection from $K$ to $G$ in Fig. 1. Here, $G$ stands for the performance on an efficiency frontier for an undesirable output. The piece-wise linear contour line $(G-H-D)$ indicates such a frontier for the undesirable output under natural disposability.

SE (South East) projections to enhance environmental performance under managerial disposability The projection from $K$ to $E$ indicates an opposite case, indicating managerial disposability. In the case, an efficiency frontier for $K$ can be found on the contour line $(D-E-F)$ where we can determine the level of unified efficiency under managerial disposability.

Lower and upper bounds on input vector As depicted in Fig. 1, it is possible for each DMU to reduce an input vector until it can reach an efficiency frontier that consists of a desirable output and an input. Thus, the efficiency frontier indicates a lower bound for input reduction. In contrast, the DMU can increase an input vector until it can reach an efficiency frontier that consists of an undesirable output and an input. Thus, the efficiency frontier indicates an upper bound for the input increase. The status shift of a DMU from inefficiency to efficiency clearly indicates a structural uniqueness of DEA environmental assessment. The conventional use of DEA does not consider such an analytical structure that is necessary for environmental assessment.

\section{Formulations under natural and managerial disposability concepts}

\subsection{Unified efficiency under natural disposability}

The previous research of Sueyoshi and Goto (2012a, b) has proposed the following radial model to measure the level of unified efficiency on the $k$ th DMU under natural disposability $(N)$ :

$$
\begin{array}{ll}
\text { Maximize } \xi+\varepsilon_{s}\left(\sum_{i=1}^{m} R_{i}^{x} d_{i}^{x-}+\sum_{r=1}^{s} R_{r}^{g} d_{r}^{g}+\sum_{f=1}^{h} R_{f}^{b} d_{f}^{b}\right) & \\
\text { s.t. } \sum_{j=1}^{n} x_{i j} \lambda_{j}+d_{i}^{x-} & =x_{i k}
\end{array}
$$

A unified efficiency score $\left(U E N_{v}^{R}\right)$ of the $k$ th DMU under natural disposability is measured by

$$
\mathrm{UEN}_{v}^{R}=1-\left[\xi^{*}+\varepsilon_{s}\left(\sum_{i=1}^{m} R_{i}^{x} d_{i}^{x-{ }^{*}}+\sum_{r=1}^{s} R_{r}^{g} d_{r}^{g^{*}}+\sum_{f=1}^{h} R_{f}^{b} d_{f}^{b^{*}}\right)\right] \text {, }
$$


where the inefficiency score $(\xi)$ and all slack variables are determined on the optimality of Model (3). Thus, the equation within the parenthesis is obtained from the optimality of Model (3). The unified efficiency under natural disposability is obtained by subtracting the level of inefficiency from unity. All the slacks are measured by

$d_{i}^{x-*}=x_{i k}-\sum_{j=1}^{n} x_{i j} \lambda_{j}^{*}, d_{r}^{g^{*}}=\sum_{j=1}^{n} g_{r j} \lambda_{j}^{*}-\left(1+\xi^{*}\right) g_{r k} \quad$ and $\quad d_{f}^{b^{*}}=\left(1-\xi^{*}\right) b_{f k}-\sum_{j=1}^{n} b_{f j} \lambda_{j}^{*}$

on the optimality of Model (3).

Model (3) has the following dual formulation:

$$
\begin{array}{cl}
\text { Minimize } & \sum_{i=1}^{m} v_{i} x_{i k}-\sum_{r=1}^{s} u_{r} g_{r k}+\sum_{f=1}^{h} w_{f} b_{f k}+\sigma \\
\text { s.t. } & \sum_{i=1}^{m} v_{i} x_{i j}-\sum_{r=1}^{s} u_{r} g_{r j}+\sum_{f=1}^{h} w_{f} b_{f j}+\sigma \geq 0 \quad(j=1, \ldots, n), \\
& \sum_{r=1}^{s} u_{r} g_{r k}+\sum_{f=1}^{h} w_{f} b_{f k}=1 \\
& v_{i} \geq \varepsilon_{s} R_{i}^{x} \quad(i=1, \ldots, m) \\
& u_{r} \geq \varepsilon_{s} R_{r}^{g} \quad(r=1, \ldots, s) \\
& w_{f} \geq \varepsilon_{s} R_{f}^{b} \quad(f=1, \ldots, h) \& \\
& \sigma: \mathrm{URS} .
\end{array}
$$

where $v_{i}(i=1, \ldots, m), u_{r}(r=1, \ldots, s)$ and $w_{f}(f=1, \ldots, h)$ are all positive dual variables (i.e., multipliers) related to the first, second and third groups of constraints in Model (3). The dual variable $(\sigma)$ is obtained from the fourth constraint of Model (3).

The comparison between Models (3) and (5) provides us with the following three concerns: First, the objective value of Model (3) equals that of Model (5) on optimality. Thus, we have the following relationship:

$\xi^{*}+\varepsilon_{s}\left[\sum_{i=1}^{m} R_{i}^{x} d_{i}^{x-*}+\sum_{r=1}^{s} R_{r}^{g} d_{r}^{g^{*}}+\sum_{f=1}^{h} R_{f}^{b} d_{f}^{b^{*}}\right]=\sum_{i=1}^{m} v_{i}^{*} x_{i k}-\sum_{r=1}^{s} u_{r}^{*} g_{r k}+\sum_{f=1}^{h} w_{f}^{*} b_{f k}+\sigma^{*}$

on optimality. A unified efficiency score $\left(\mathrm{UEN}_{v}^{R}\right)$ of the $k$ th DMU under natural disposability is also measured by

$$
\mathrm{UEN}_{v}^{R}=1-\left(\sum_{i=1}^{m} v_{i}^{*} x_{i k}-\sum_{r=1}^{s} u_{r}^{*} g_{r k}+\sum_{f=1}^{h} w_{f}^{*} b_{f k}+\sigma^{*}\right) .
$$

Second, an important feature of Model (3) is that all dual variables are positive as formulated in Model (5). Thus, information on the three groups of production factors is fully utilized in the assessment of Model (3). Finally, each dual variable indicates an amount of change in unified inefficiency under natural disposability due to a unit change in the corresponding production factor. 


\subsection{Unified efficiency under managerial disposability}

Shifting our research interest from natural disposability to managerial disposability $(M)$, where the first priority is environmental performance and the second priority is operational performance, this study utilizes the following radial model that measures the unified efficiency of the $k$ th DMU under managerial disposability (Sueyoshi and Goto, 2012b):

$$
\begin{array}{ll}
\text { Maximize } \xi+\varepsilon_{s}\left(\sum_{i=1}^{m} R_{i}^{x} d_{i}^{x+}+\sum_{r=1}^{s} R_{r}^{g} d_{r}^{g}+\sum_{f=1}^{h} R_{f}^{b} d_{f}^{b}\right) \\
\text { s.t. } \quad \sum_{j=1}^{n} x_{i j} \lambda_{j}-d_{i}^{x+}=x_{i k} \quad(i=1, \ldots, m), \\
& \sum_{j=1}^{n} g_{r j} \lambda_{j}-d_{r}^{g}-\xi g_{r k}=g_{r k} \quad(r=1, \ldots, s) \\
& \sum_{j=1}^{n} b_{f j} \lambda_{j}+d_{f}^{b}+\xi b_{f k}=b_{f k} \quad(f=1, \ldots, h), \\
& \sum_{j=1}^{n} \lambda_{j}=1, \\
& \lambda_{j} \geq 0(j=1, \ldots, n), \xi: U R S, d_{i}^{x+} \geq 0 \quad(i=1, \ldots, m), \\
& d_{r}^{g} \geq 0 \quad(r=1, \ldots, s) \& d_{f}^{b} \geq 0 \quad(f=1, \ldots, h) .
\end{array}
$$

An important feature of Model (8) is that it changes $+d_{i}^{x-}$ of Model (3) to $-d_{i}^{x+}$ in order to attain the status of managerial disposability. A unified efficiency score $\left(U E M_{v}^{R}\right)$ on the $k$ th DMU under managerial disposability is measured by

$$
\mathrm{UEM}_{v}^{R}=1-\left[\xi^{*}+\varepsilon\left(\sum_{i=1}^{m} R_{i}^{x} d_{i}^{x+*}+\sum_{r=1}^{s} R_{r}^{g} d_{r}^{g^{*}}+\sum_{f=1}^{h} R_{f}^{b} d_{f}^{b^{*}}\right)\right]
$$

where the inefficiency score and all slacks are determined on the optimality of Model (8). Thus, the equation within the parenthesis, obtained from the optimality of Model (8), indicates the level of unified inefficiency under managerial disposability. The unified efficiency is obtained by subtracting the level of inefficiency from unity.

Model (8) has the following dual formulation:

$$
\begin{aligned}
& \text { Minimize }-\sum_{i=1}^{m} v_{i} x_{i k}-\sum_{r=1}^{s} u_{r} g_{r k}+\sum_{f=1}^{h} w_{f} b_{f k}+\sigma \\
& \text { s.t. }-\sum_{i=1}^{m} v_{i} x_{i j}-\sum_{r=1}^{s} u_{r} g_{r j}+\sum_{f=1}^{h} w_{f} b_{f j}+\sigma \geq 0(j=1, \ldots, n), \\
& \quad \sum_{r=1}^{s} u_{r} g_{r k}+\sum_{f=1}^{h} w_{f} b_{f k}=1, \\
& v_{i} \geq \varepsilon_{s} R_{i}^{x}(i=1, \ldots, m), \\
& u_{r} \geq \varepsilon_{s} R_{r}^{g}(r=1, \ldots, s), \\
& w_{f} \geq \varepsilon_{s} R_{f}^{b}(f=1, \ldots, h) \& \\
& \sigma: \mathrm{URS},
\end{aligned}
$$


where $v_{i}(i=1, \ldots, m), u_{r}(r=1, \ldots, s)$ and $w_{f}(f=1, \ldots, h)$ are all dual variables related to the first, second and third groups of constraints in Model (8). The dual variable $(\sigma)$ is obtained from the fourth constraint of Model (8). The objective value of Model (8) equals that of Model (10) on optimality. Consequently, we have the following relationship on optimality:

$\xi^{*}+\varepsilon_{s}\left[\sum_{i=1}^{m} R_{i}^{x} d_{i}^{x+*}+\sum_{r=1}^{s} R_{r}^{g} d_{r}^{g^{*}}+\sum_{f=1}^{h} R_{f}^{b} d_{f}^{b^{*}}\right]=-\sum_{i=1}^{m} v_{i}^{*} x_{i k}-\sum_{r=1}^{s} u_{r}^{*} g_{r k}+\sum_{f=1}^{h} w_{f}^{*} b_{f k}+\sigma^{*}$

A unified efficiency score $\left(U E M_{v}^{R}\right)$ of the $k$ th DMU under managerial disposability is also measured by

$$
\mathrm{UEM}_{v}^{R}=1-\left(-\sum_{i=1}^{m} v_{i}^{*} x_{i k}-\sum_{r=1}^{s} u_{r}^{*} g_{r k}+\sum_{f=1}^{h} w_{f}^{*} b_{f k}+\sigma^{*}\right) .
$$

The difference between Models (5) and (10) is that the former model has $\sum_{i=1}^{m} v_{i} x_{i k}$ in the objective function and $\sum_{i=1}^{m} v_{i} x_{i j}$ for $j=1, \ldots, n$ in the constraints, while the latter model has $-\sum_{i=1}^{m} v_{i} x_{i k}$ in the objective function and $-\sum_{i=1}^{m} v_{i} x_{i j}$ for $j=1, \ldots, n$ in the constraints. No other difference exists between them. The previous description on dual variables on Model (5) can be applied to those of Model (10).

\section{Possible Occurrence of Desirable Congestion (Eco-technology Innovation)}

A possible occurrence of Undesirable Congestion (UC) and Desirable Congestion (DC) can be incorporated into Eqs. (1) and (2) after slightly modifying output vectors and an input vector, respectively, as follows:

$$
\begin{aligned}
& P_{U C}^{N}(X)=\left\{(G, B): G \leq \sum_{j=1}^{n} G_{j} \lambda_{j}, B=\sum_{j=1}^{n} B_{j} \lambda_{j}, X \geq \sum_{j=1}^{n} X_{j} \lambda_{j}, \sum_{j=1}^{n} \lambda_{j}=1 \& \lambda_{j} \geq 0 \quad(j=1, \ldots, n)\right\} \& \\
& P_{D C}^{M}(X)=\left\{(G, B): G=\sum_{j=1}^{n} G_{j} \lambda_{j}, B \geq \sum_{j=1}^{n} B_{j} \lambda_{j}, X \leq \sum_{j=1}^{n} X_{j} \lambda_{j}, \sum_{j=1}^{n} \lambda_{j}=1 \& \lambda_{j} \geq 0 \quad(j=1, \ldots, n)\right\} .
\end{aligned}
$$

Equation (13) incorporates a possible occurrence of UC under natural disposability by assigning equality on $B$. Equation (14) incorporates a possible occurrence of DC under managerial disposability by assigning equality on $G$. The difference between the two equations can be found on the allocation of the equality sign on desirable or undesirable outputs. See previous research works such as Sueyoshi and Goto (2012c, d, e, 2013) on a possible occurrence on UC and DC.

Desirable congestion (eco-technology innovation) Since we are interested in identifying an occurrence of Desirable Congestion (DC) under managerial disposability, this study pays attention to Eq. (14). To examine such a possible occurrence, this study proposes the following model that maintains equality constraints (so, no slack variable) on desirable outputs, as specified in Eq. (14): 


$$
\begin{aligned}
\text { Maximize } & \xi+\varepsilon_{s}\left[\sum_{i=1}^{m} R_{i}^{x} d_{i}^{x+}+\sum_{f=1}^{h} R_{f}^{b} d_{f}^{b}\right] \\
\text { s.t. } \quad & \sum_{j=1}^{n} x_{i j} \lambda_{j}-d_{i}^{x+}=x_{i k} \quad(i=1, \ldots, m), \\
& \sum_{j=1}^{n} g_{r j} \lambda_{j}-\xi g_{r k}=g_{r k} \quad(r=1, \ldots, s) \\
& \sum_{j=1}^{n} b_{f j} \lambda_{j}+d_{f}^{b}+\xi b_{f k}=b_{f k} \quad(f=1, \ldots, h), \\
& \sum_{j=1}^{n} \lambda_{j}=1, \\
& \lambda_{j} \geq 0(j=1, \ldots, n), \xi: U R S, d_{i}^{x+} \geq 0 \quad(i=1, \ldots, m), \\
& \text { and } d_{f}^{b} \geq 0 \quad(f=1, \ldots, h) .
\end{aligned}
$$

Model (15) drops slack variables related to desirable outputs so that they are considered as equality constraints. The other groups of constraints on inputs and undesirable outputs maintain slacks so that they can be considered as inequality constraints. For example, $\sum_{j=1}^{n} x_{i j} \lambda_{j}-d_{i}^{x+}=x_{i k}$ is equivalent to $\sum_{j=1}^{n} x_{i j} \lambda_{j} \geq x_{i k}$ for all $i$. The description on input slacks is also applicable to undesirable outputs.

Model (15) has the following dual formulation:

$$
\begin{aligned}
& \text { Minimize }-\sum_{i=1}^{m} v_{i} x_{i k}-\sum_{r=1}^{s} u_{r} g_{r k}+\sum_{f=1}^{h} w_{f} b_{f k}+\sigma \\
& \text { s.t. }-\sum_{i=1}^{m} v_{i} x_{i j}-\sum_{r=1}^{s} u_{r} g_{r j}+\sum_{f=1}^{h} w_{f} b_{f j}+\sigma \geq 0 \quad(j=1, \ldots, n) \text {, } \\
& \sum_{r=1}^{s} u_{r} g_{r k}+\sum_{f=1}^{h} w_{f} b_{f k}=1, \\
& v_{i} \geq \varepsilon_{s} R_{i}^{x}(i=1, \ldots, m), \\
& u_{r} \text { : URS }(r=1, \ldots, s) \text {, } \\
& w_{f} \geq \varepsilon_{s} R_{f}^{d} \quad(f=1, \ldots, h) \quad \& \\
& \sigma: \text { URS. }
\end{aligned}
$$

An important feature of Model (16) is that the dual variables $\left(u_{r}\right.$ : URS for $r=1, \ldots$, $s)$ are unrestricted in their signs because Model (16) drops slack variables related to the desirable outputs.

A unified efficiency score (UEM) of the $k$ th DMU under managerial disposability is measured by

$$
\mathrm{UEM}=1-\left[\xi^{*}+\varepsilon_{s}\left(\sum_{i=1}^{m} R_{i}^{x} d_{i}^{x+*}+\sum_{f=1}^{h} R_{f}^{b} b_{f}^{b^{*}}\right)\right]=1-\left[-\sum_{i=1}^{m} v_{i}^{*} x_{i k}-\sum_{r=1}^{s} u_{r}^{*} g_{r k}+\sum_{f=1}^{h} w_{f}^{*} b_{f k}+\sigma^{*}\right],
$$


where all variables are determined on the optimality of Models (15) and (16). The equation within the parenthesis, obtained from the optimality of Models (15) and (16), indicates the level of unified inefficiency under managerial disposability. The unified efficiency is obtained by subtracting the level of inefficiency from unity.

\subsection{Occurrence of DC (Eco-technology Innovation)}

After solving Model (16), this study can identify an occurrence of DC, or green technology innovation, by the following rule along with the assumption on a unique optimal solution (i.e., unique projection and a unique reference set):

(a) if $u_{r}^{*}<0$ for some (at least one) $r$, then "strong DC" occurs on the $k$ th DMU,

(b) if $u_{r}^{*}>0$ for all $r$, then "no DC" occurs on the $k$ th DMU and

(c) In the others, including $u_{r}^{*}=0$ for some (at least one) $f$, then "weak DC" occurs on the $k$ th DMU.

Note that if $u_{r}^{*}<0$ for some $r$ and $u_{r^{\prime}}^{*}=0$ for the other $r^{\prime}$, then the weak and strong DCs coexist on the $k$ th DMU. This study considers it as the strong DC, so indicating technology innovation on undesirable outputs. It is important to note that $u_{r}^{*}<0$ for all $\mathrm{r}$ is the best case because an increase in any desirable output always decreases an amount of undesirable outputs. Meanwhile, if $u_{r}^{*}<0$ is identified for some $r$, then it indicates that there is a chance to reduce an amount of undesirable output(s). Therefore, this study considers the second case as an occurrence of DC.

\subsection{Damage elasticity}

Before describing the measurement of DTR under DC, let us consider a simple case where three production factors have a single component. Let us consider a supporting hyperplane that is specified by $-v^{*} x-u^{*} g+w^{*} b+\sigma^{*}=0$, where $u^{*}$ and $\sigma^{*}$ are unrestricted in their signs and the other parameters are strictly positive on optimality. See the objective function of Eq. (16). The supporting hyperplane becomes $b=\left(v^{*} x+u^{*} g-\sigma^{*}\right) / w^{*}$. The slope of the supporting hyperplane is determined by the sign of $u$.

The status of no DC $\left(u^{*}>0\right)$ is further classified into the three cases. To describe the classification, let us take the marginal rate of change and the average change of the supporting hyperplane. They become $\frac{d b}{d g}=\frac{u^{*}}{w^{*}}$ and $\frac{b}{g}=\frac{u^{*}}{w^{*}}-\frac{\sigma^{*}-v^{*} x}{w^{*} g}$, respectively. Consequently, the scale elasticity related to an undesirable output is measured by $e_{b}=\left(\frac{d b}{d g}\right) /\left(\frac{b}{g}\right)=1 /\left(1-\frac{\sigma^{*}-v^{*} x}{u^{*} g}\right)$. The scale elasticity $\left(e_{b}\right)$ and the type of DTR are determined by $\sigma$ and $u$ as follows:
(a) $e_{b}>1 \leftrightarrow$ Increasing DTR $\leftrightarrow \sigma^{*}-v^{*} x>0$
(b) $e_{b}=1 \leftrightarrow$ Constand DTR $\leftrightarrow \sigma^{*}-v^{*} x=0$ and
(c) $e_{b}<1 \leftrightarrow$ Decresing DTR $\leftrightarrow \sigma^{*}-v^{*} x<0$.

\subsection{Damage economies}

If the $k$ th DMU is efficient in unified performance under managerial disposability, then the degree of Damage Economies (DE) for multiple components, corresponding to damage elasticity $\left(e_{b}\right)$, can be determined by 


$$
\begin{aligned}
\mathrm{DE} & =\left(\sum_{r=1}^{s} u_{r}^{*} g_{r k}\right) /\left(\sum_{f=1}^{h} w_{f}^{*} b_{f k}\right)=\left(\sum_{r=1}^{s} u_{r}^{*} g_{r k}\right) /\left(\sum_{i=1}^{m} v_{i}^{*} x_{i k}+\sum_{r=1}^{s} u_{r}^{*} g_{r k}-\sigma^{*}\right) \\
& =1 /\left(1-\left(\sigma^{*}-\sum_{i=1}^{m} v_{i}^{*} x_{i k}\right) /\left(\sum_{r=1}^{s} u_{r}^{*} g_{r k}\right)\right) \\
& =1 /\left(1-\left(\sigma^{*}-\zeta_{k}^{x^{*}}\right) /\left(\sum_{r=1}^{s} u_{r}^{*} g_{r k}\right)\right)
\end{aligned}
$$

where $\zeta_{k}^{x^{*}}=\sum_{i=1}^{m} v_{i}^{*} x_{i k}$. The input-related variable implies an influence level from inputs under managerial disposability on the degree of $D E$. Equation (19) incorporates $-\sum_{i=1}^{m} v_{i}^{*} x_{i k}-\sum_{r=1}^{s} u_{r}^{*} g_{r k}+\sum_{f=1}^{h} w_{f}^{*} b_{f k}+\sigma^{*}=0$ in the reformulation, indicating the degree of inefficiency.

Meanwhile, if the $k$ th DMU is inefficient in unified performance under managerial disposability, then the inefficient DMU needs to be projected onto an efficiency frontier. In the case, the degree of $D E$ can be determined by

$$
\begin{aligned}
\mathrm{DE} & =\left(\sum_{r=1}^{s} u_{r}^{*} g_{r k}\right) /\left(\sum_{f=1}^{h} w_{f}^{*}\left(b_{f k}-d_{f}^{b^{*}}\right)\right) \\
& =\left(\sum_{r=1}^{s} u_{r}^{*} g_{r k}\right) /\left(\sum_{f=1}^{h}\left(w_{f}^{*} b_{r k}-w_{f}^{*} d_{f}^{b^{*}}\right)\right) \\
& =\left(\sum_{r=1}^{s} u_{r}^{*} g_{r k}\right) /\left(\sum_{i=1}^{m} v_{i}^{*}\left(x_{i k}+d_{i}^{x+*}\right)+\sum_{r=1}^{s} u_{r}^{*} g_{r k}-\sigma^{*}\right) \\
& =1 /\left(1+\left(\left(\sum_{i=1}^{m} v_{i}^{*}\left(x_{i k}+d_{i}^{x++^{*}}\right)-\sigma^{*}\right) / \sum_{r=1}^{s} u_{r}^{*} g_{r k}\right)\right) \\
& =1 /\left(1-\left(\left(\sigma^{*}-\sum_{i=1}^{m} v_{i}^{*} x_{i k}\right) / \sum_{r=1}^{s} u_{r}^{*} g_{r k}\right)\right) \\
& =1 /\left(1-\left(\left(\sigma^{*}-\zeta_{k}^{x^{*}}\right) / \sum_{r=1}^{s} u_{r}^{*} g_{r k}\right)\right) .
\end{aligned}
$$

Equation (20) incorporates $\xi^{*}+\varepsilon_{s}\left(\sum_{i=1}^{m} R_{i}^{x} d_{i}^{x+*}+\sum_{f=1}^{h} R_{f}^{b} d_{f}^{b^{*}}\right)=-\sum_{i=1}^{m} v_{i}^{*} x_{i k}$ $-\sum_{r=1}^{s} u_{r}^{*} g_{r k}+\sum_{f=1}^{h} w_{f}^{*} b_{f k}+\sigma^{*}$. The reformulation (20) has the following three assumptions in the reformulation. First, after the inefficient DMU is projected onto an efficiency frontier, it has $\xi^{*}=0$. Second, the existence of positive slacks on all production factors so that $v_{i}^{*}=\varepsilon_{s} R_{i}^{x}$ for all $\mathrm{i}$ and $w_{f}^{*}=\varepsilon_{s} R_{f}^{b}$ for all $\mathrm{f}$ because of complementary slackness conditions of linear programming. Second, the observed inputs are larger than their slacks so that their differences are positive. Finally, Eq. (20) considers $\zeta_{k}^{x^{*}}=\sum_{i=1}^{m} v_{i}^{*}\left(x_{i k}+d_{i}^{x+^{*}}\right) \cong \sum_{i=1}^{m} v_{i}^{*} x_{i k}$ because this study is interested in the type of DTR on an efficient frontier that is mainly determined by desirable and undesirable outputs. Hence, $d_{i}^{x+{ }^{*}} \cong 0$ is assumed for all $f$ for our computational tractability, indicating that all slacks related to inputs are positive but very close to zero on the efficiency frontier. 


\subsection{Applications of the proposed approach}

Applications of the proposed approach can be found in the following articles:

(a) Sueyoshi and Goto (2016a).

(b)Sueyoshi and Yuan (2016b).

Therefore, this study does not document an application of the proposed approach, here, rather discussing their theoretical foundations, as discussed the preceding sections.

\section{Conclusion and future extensions}

To measure the level of sustainability regarding various organizations in public and private sectors, this study discussed the importance regarding the use of DEA for environmental assessment. The proposed environmental assessment provided policy makers and corporate leaders with not only the level of sustainability but also information regarding how to invest for technology innovation for abatement of undesirable outputs. A contribution of this study is to convey a message that DEA is a methodology for not only efficiency measurement but also identifying eco-technology innovation. Such a view was discussed in this research.

The proposed DEA approach has three drawbacks as a methodology for environmental assessment. First, the proposed DEA approach assumes that all unified efficiency measures are uniquely determined on optimality. If the assumption is dropped, the proposed radial models need to incorporate strong complementary slackness conditions into their formulations to obtain a unique optimal solution. Second, this study can incorporate a time horizon into the computational framework of DEA environmental assessment. Consequently, the extension makes it possible to handle a time series data set on environmental assessment. Finally, this study does not discuss analytical features on RTS, DTS and DTR. The mathematical issue will be discussed in another article as an extension of this study.

Competing interests

This study is not intersted in a computational concern. Therefore, this study discusses a theoretical aspect on DTR, or ecotechnology innvation, within the framework of DEA environmental asessment.

\section{Publisher's Note}

Springer Nature remains neutral with regard to jurisdictional claims in published maps and institutional affiliations.

Received: 20 February 2017 Accepted: 21 March 2017

Published online: 04 April 2017

References

Glover F, Sueyoshi T (2009) Contributions of Professor William W. Cooper in operations research and management science. Eur J Oper Res 197:1-16

Ijiri Y, Sueyoshi T (2010) Accounting essays by Professor William W. Cooper: revisiting in commemoration of his 95th birthday. ABACUS J Account Financ Bus Stud 46:464-505

Porter M, van der Linde C (1995) Toward a new conception of the environment-competitiveness relationship. J Econ Perspect 9:97-118

Sueyoshi T, Goto M (2012a) Data Envelopment Analysis for environmental assessment: comparison between public and private ownership in petroleum industry. Eur J Oper Res 216:668-678

Sueyoshi T, Goto M (2012b) DEA radial measurement for environmental assessment and planning: desirable procedures to evaluate fossil fuel power plants. Energy Policy 41:422-432 
Sueyoshi T, Goto M (2012c) Returns to scale and damages to scale under natural and managerial disposability: strategy, efficiency and competitiveness of petroleum firms. Energy Econ 34:645-662

Sueyoshi T, Goto M (2012d) Returns to scale, damages of scale, marginal rate of transformation and rate of substitution in DEA environmental assessment. Energy Econ 34:905-917

Sueyoshi T, Goto M (2012e) Returns to scale and damages to scale on U.S. fossil fuel power plants: radial and non-radial approaches for DEA environment assessment. Energy Econ 34:2240-2259

Sueyoshi T, Goto M (2013) Returns to scale vs. damages to scale in Data Envelopment Analysis: an impact of U.S. Clean Air Act on coal-fired power plants. OMEGA 41:164-175

Sueyoshi T, Goto M (2016) Undesirable congestion under natural disposability and desirable congestion under managerial disposability in U.S. electric power industry measured by DEA environmental assessment. Energy Econ 55:173-188

Sueyoshi T, Goto M (2017) Environmental assessment on energy and sustainability by data envelopment analysis. Wiley, London (forthcoming)

Sueyoshi T, Yuan Y (2016a) Returns to damage under undesirable congestion and damages to return under desirable congestion measured by DEA envernmental assessment with multiplier restriction: economic and energy planning for social sustainability in China. Energy Econ 56:288-309

Sueyoshi T, Yuan Y (2016b) Chapter 17: DEA Environmental Assessment (II): a literature study. In: Hwang et al (eds) Handbook of Operations Analytics using Data Envelopment Analysis, international series of operations research \& management science, vol 239. Springer, New York, pp 445-481

Sueyoshi T, Yuan Y, Goto M (2017) A literature survey for DEA environmental assessment on energy and sustainability. Energy Econ 62:104-124

\section{Submit your manuscript to a SpringerOpen ${ }^{\circ}$ journal and benefit from:}

- Convenient online submission

\section{- Rigorous peer review}

- Immediate publication on acceptance

- Open access: articles freely available online

- High visibility within the field

- Retaining the copyright to your article 